\title{
Dispensing patterns of drugs used for neuropathic pain and adherence to NeuPSIG guideline: an observational study
}

\author{
Bahia Chahine ${ }^{*}$ (ID) and Farah Al Souheil
}

\begin{abstract}
Background: Neuropathic pain is a common chronic ailment that can be challenging to treat because of the heterogeneity of its etiologies, symptoms, and underlying mechanisms. Drugs to treat neuropathic pain are highly prescribed; however, limited data exist on the rates and patterns of dispensing in Lebanon. This study was conducted to assess the patterns of dispensing neuropathic pain drugs and adherence to NeuPSIG guideline in the Lebanese adult population. An observational study was conducted at 30 community pharmacies over 10 months. Adults presenting to the community pharmacy with a prescription of at least one pain medication and diagnosed with a neuropathic pain disorder were interviewed using a questionnaire that included demographic characteristics of patients, comorbidities, neuropathic pain disorder type, physicians' specialty, and the class and number of prescribed medications.

Results: A total of 360 patients diagnosed with painful neuropathy were enrolled in our study. The mean patients' age was 50.2 years. Guideline-recommended first-line agents (serotonin-norepinephrine reuptake inhibitors, tricyclic antidepressants, and gabapentinoids) were prescribed to 278 patients (77.2\%), with pregabalin being the most used drug (60.5\%), nonsteroidal anti-inflammatory drugs (46.8\%), opioids (15.8\%), and topical agents (5.5\%). Combination treatment for neuropathic pain was prescribed to $78.3 \%$ of patients.
\end{abstract}

Conclusions: In Lebanon, neuropathic pain management comply with the international NeuPSIG guideline. Pregabalin is the most commonly used drug, and combination treatments are usually needed to alleviate neuropathic pain.

Keywords: Neuropathic pain, Medication use, Community pharmacy, Pregabalin, Gabapentinoids

\section{Background}

Pain is an unpleasant sensation that affects a person's physiological and emotional health, socioeconomic well-being, and quality of life [1]. Neuropathic pain $(\mathrm{NeP})$ is described as "pain caused by a lesion or disorder of the somatosensory nervous system," which may be central or peripheral, according to the International Association for the Study of Pain [1]. The prevalence of $\mathrm{NeP}$ disorders in Lebanon is undetermined; however, it could be as high as $7 \%$ to $9 \%$ in the general population [2]. $\mathrm{NeP}$ is very challenging to manage because of the

*Correspondence: bahia.chahine@liu.edu.lb

School of Pharmacy, Lebanese International University, Beirut, Lebanon heterogeneity of the etiologies, symptoms, and underlying mechanisms; therefore, management focuses on pain medications rather than disease-modifying treatments [3]. In Lebanon, the Lebanese society of study of pain works per recommendations provided by the International Association of the Study of Pain (IASP). Recommended first-line treatments for NeP syndromes by the IASP/ Neuropathic Pain Special Interest Group (NeuPSIG) include gabapentinoids (pregabalin and gabapentin), serotonin-norepinephrine reuptake inhibitors (SNRIs) (duloxetine and venlafaxine), and tricyclic antidepressants (TCAs) (amitriptyline and nortriptyline) $[4,5]$. Such recommendation is also endorsed by other pain management societies, namely, the Canadian Pain Society (CPS), the European Federation of 
Neurological Sciences (EFNS), and the American Academy of Neurology (AAN).

Furthermore, tramadol is considered as a second-line drug by most guidelines $[4,6,7]$. The more potent opioids are regarded as a third-line agent by NeuPSIG and EFNS [7]. The use of opioids is limited for short-term treatment of $\mathrm{NeP}$ given their limited benefits compared to imposed risks in case of chronic use. Downsides to using opioids include an increased risk of withdrawal, overdose, dependency, addiction, and death [5]. Finally, NeuPSIG recommends alternative second-line options as capsaicin and lidocaine patches for localized effects in peripheral neuropathy and botulinum toxin as a third line [4]. Until now, no sufficient evidence is present to support or refute the suggestion that oral nonsteroidal anti-inflammatory drugs (NSAIDs) effectively treat neuropathic pain [8].

Driven by the fact that guidelines targeting neuropathy must be region-specific due to possible ethnic variations in genetics, etiology, and severity of pain, an Arab meeting was recently held [9]. Recommendations set in the meeting compromised the preference of gabapentinoids and TCAs (secondary amines in specific, nortriptyline, and desipramine) over SNRIs and opioids (controlled release) [9].

Given that $\mathrm{NeP}$ is often not effectively controlled with a single agent, NeuPSIG guidelines recommend using combinations in patients inadequately relieved by a single agent, except for the elderly $[4,5]$. Combinations include Gabapentinoid and TCA or SNRI [4].

Non-pharmacological options are also available; however, they received a weak recommendation from NeuPSIG. Those measures include epidural injection of corticosteroids; pulsed radiofrequency that modulates synaptic transmission by targeting the electrical field; adhesiolysis; sympathetic blockade with a local anesthetic; radiofrequency denervation, which causes nerve ablation using heat, and spinal cord stimulation [4]. Recently, several clinics providing such services have been established in Lebanon.

However, despite the significant advances in this field, a study recently conducted in Lebanon by Zeitoun and colleagues found a lack of a well-structured system for pain management [10]. In this regard, one of the chief recommendations suggested by the American Pain Society (APS) for an enhanced pain management practice is the ongoing assessment of the quality of practice [11]. That is the actual life implementation of updated guidelines. Previous studies have also shown an increased pain severity and degraded quality of life of middle-eastern, neuropathic pain patients and the soaring rates of $\mathrm{NeP}$ in both diabetes and chronic low-back pain in the region [9]. Given the lack of previous research that examines the dispensing pattern of $\mathrm{NeP}$ medications in Lebanon, there was a pressing need to conduct this study.

The main objective was to assess the pattern of medications dispensing to adult patients diagnosed with neuropathic pain disorders and the association between patient factors and the choice of medication subgroup through interviewing patients in a community pharmacy setting.

\section{Methods}

This prospective cross-sectional study was conducted between January and October 2019 in a communitybased pharmacy setting in Lebanon, and gathered data from 360 adult patients having a neuropathic pain disorder. The study was approved by the Lebanese International University Institutional Review Board (2018/10). As our research did not include any active intervention, we obtained only oral consent from the patients. Consent was also acquired from all chief pharmacists for the research to be conducted on their premises.

A list of all 3000 pharmacies in Lebanon including name, address, and contact information was provided by the order of Lebanese pharmacists (OPL). The list was transferred to an Excel sheet, randomized using SPSS (statistical package for social science) version 23 , and a proportionate sample of 30 pharmacies was selected from all Lebanese districts (Beirut, Mount Lebanon, North, South, and Beqaa). A sample of 355 patients was targeted to allow for adequate power for multivariable analyses to be carried out based on a population size of five million inhabitants in Lebanon, a 9\% expected prevalence of $\mathrm{NeP}$ disorders and a $99.9 \%$ confidence level $[2,12]$.

Consecutive customers arriving at community pharmacies seeking pain medication (Gabapentinoids, SNRIs, TCAs, NSAIDs, tramadol, topical capsaicin, or topical lidocaine) were approached by the researcher for potential enrollment in the study. It is worth noting that gabapentinoids and tramadol may only be obtained with a doctor's prescription. However, while the Ministry of Public Health continually inspects tramadol dispense records, no clear regulations to control gabapentinoids dispensing. That is, the latter may be dispensed by pharmacists without a prescription [13].

After giving a verbal consent to participate, patients were asked about the type of pain experienced. Subjects were primarily included in the study if they were (1) 18 years and older, (2) clinically diagnosed with a neuropathic pain disorder (cervical or lumbar radiculopathy, diabetic neuropathy, spinal cord injury, post-herpetic neuralgia, postoperative neuropathic pain, post-traumatic neuralgia, trigeminal neuralgia, atypical facial pain, multiple sclerosis, stroke leading to central post-stroke pain) by an orthopedist, neurologist, or neurosurgeon, (3) taking at least one pain medication, and (4) willing to 
participate in our study. Patients were excluded if they (1) were not able to provide the name and contact information of the prescribing physician or (2) had a concomitant pain unrelated to the study-defined condition such as cancer, hip fracture, and nociceptive pain that would interfere with the assessment of neuropathic pain and the selection of pain medications.

The questionnaire was first piloted on a sample of 20 patients from four different pharmacies across Lebanon for 3 days/ pharmacy to ensure the appropriateness of questions and to foresee the sufficient time interval needed to be spent at each location. Data were then discarded and were never included in the primary analysis, and time spent at each community pharmacy was increased to an entire ' per pharmacy. Eligible patients were then interviewed face-to-face in the pharmacy waiting area. Data were filled by the researcher using a structured questionnaire that was translated into Arabic language and consisted of 25-items considering; sociodemographic characteristics of patients, comorbidities, neuropathic pain disorder type, and physicians' specialty and contact information. Such characteristics may affect the choice of therapy and the favor of a second-line agent over other first-line options for instance, alcoholism may accentuate CNS depression caused by gabapentinoids and opioids; level of education and socioeconomic status may affect the level of acceptance or avoidance of being prescribed a certain medication or may decide the characteristics of the prescribing physician. Finally, we requested each patient's permission to obtain confirmatory information about the diagnosis from the physician by telephone, who was informed of the research objective for a legitimate process.

Patients were also asked to report any other currently used analgesic if the latter is not listed in the prescription and were asked to justify its use. It is worth mentioning that tramadol was the only opioid included in the questionnaire, because all other opioids dispensary is restricted to pharmacies enrolled in a particular program (MPH213-1) and thus may not be available in all Lebanese pharmacies.

Data collected from questionnaires were recorded and analyzed using IBM SPSS Statistics for Windows, Version 23.0, 2019 (Armonk, NY: IBM Corp). Descriptive statistics were calculated for all study variables, including means and standard deviations (SD) for continuous measures, counts, and percentages for categorical variables. Separate multivariable logistic regression models were used to evaluate the association between patientlevel variables and the dependent variables of opioid medication use, first-line guideline-recommended medication use, and NSAIDs use. Statistical significance was set at a $p$ value of less than 0.05 .

\section{Results}

Nearly 3420 customers were approached, of which 427 were diagnosed with neuropathic pain disorder and were eligible to participate in this study. The response rate was $84.3 \%$; 427 patients approached, 360 agreed to participate. The mean patients' age was 50.2 years with a range of 23-88 years, and 55\% were females. Of the 360 patients, 110 (30.6\%) had diabetes mellitus, 108 (30\%) had anxiety, and $24(9.4 \%)$ had depression (Table 1$)$. Level of education and socioeconomic level were not associated with a specific prescribing pattern.

The most common painful neuropathic disorder encountered was cervical or lumbar radiculopathy 154 (42.8\%), as shown in Figure 1. Guideline-recommended first-line agents were prescribed in 278 (77.2\%) of patients. NSAIDs were used by 169 (46.8\%) and opioids by $57(15.8 \%)$ of patients. Monotherapy for neuropathic pain was prescribed to $78(21.7 \%)$ of the patients (Figure 2). Moreover, polypharmacy in pain was reported in $282(78.3 \%)$ of the patients, and the most common dual combination used was pregabalin plus NSAIDs (21.6\%).

Physicians were involved in the medication plan of 300 patient $(83.5 \%)$, of which $50.5 \%$ were followed up by an orthopedist, $26.6 \%$ by a neurologist, and $6.1 \%$ by a neurosurgeon. Patients who received pain medication without a physician's recommendation accounted for $16.5 \%$. Of the neuropathic pain etiologies, post-herpetic neuralgia patients reported the lowest rate of physicians prescribed pain medication use, $41 \%$.

Table 2 summarizes the results of the multivariate analysis. Guideline-recommended first-line medication utilization was associated with physician visits, whereas NSAIDs utilization was not. Older patients experienced significantly less initiation of first-line medications. Opioids were significantly less initiated in patients with diabetic neuropathy and cervical or lumbar radiculopathy. However, they were more significantly used in patients with positive alcohol intake and smoking history.

\section{Discussion}

It is essential for societies projecting guidelines to constantly assess the consistency of physicians' prescriptions with the set recommendations. Several studies conducted worldwide have focused on the epidemiology and treatment of neuropathic pain. However, none was performed in the Lebanese community. Our study was conducted across all Lebanese governorates to review the pattern of drug dispensing in patients with neuropathic pain in a community setting.

Most patients were suffering from neuropathic back pain or diabetic neuropathy. Those same predominating $\mathrm{NeP}$ types are also observed in the middle east region [9]. Furthermore, it was noted that the majority 
Table 1 Demographic and clinical characteristics

\section{Demographics and clinical variables $(N, \%)$ unless otherwise specified}

\begin{tabular}{|c|c|}
\hline Age (mean, SD) & $50.22(14.17)$ \\
\hline Female & $198(55)$ \\
\hline \multicolumn{2}{|l|}{ Marital status } \\
\hline Single & $64(17.8)$ \\
\hline Married & $296(82.2)$ \\
\hline Current smoker & $250(69.4)$ \\
\hline \multicolumn{2}{|l|}{ Alcohol consumption } \\
\hline Abstainer & $256(71.1)$ \\
\hline Drinker & $102(28.3)$ \\
\hline \multicolumn{2}{|l|}{ Lebanese governorate } \\
\hline Beirut & $126(35)$ \\
\hline Bekaa & $93(25.8)$ \\
\hline Mount Lebanon & $85(23.6)$ \\
\hline North Lebanon & $27(7.5)$ \\
\hline Nabatiye & $15(4.2)$ \\
\hline South Lebanon & $14(3.9)$ \\
\hline \multicolumn{2}{|l|}{ Comorbidities } \\
\hline Diabetes mellitus & $110(30.6)$ \\
\hline Anxiety & $108(30)$ \\
\hline Depression & $24(9.4)$ \\
\hline \multicolumn{2}{|l|}{ First-line agents } \\
\hline Any & $278(77.2)$ \\
\hline Pregabalin & $218(60.5)$ \\
\hline Gabapentin & $48(13.3)$ \\
\hline Tricyclic antidepressants & $6(1.7)$ \\
\hline Duloxetine & $4(1.1)$ \\
\hline Venlafaxine & $2(0.6)$ \\
\hline \multicolumn{2}{|l|}{ Second-line agents } \\
\hline Tramadol & $57(15.8)$ \\
\hline \multicolumn{2}{|l|}{ Other agents } \\
\hline NSAIDs & $169(46.8)$ \\
\hline Topical agents (Capsaicin, Lidocaine) & $20(5.5)$ \\
\hline \multicolumn{2}{|c|}{ The most common drug combination used to treat neuropathic pain disorders } \\
\hline Dual: Gabapentinoids + NSAIDS & $78(21.6)$ \\
\hline Gabapentinoids + Tramadol & $32(8.9)$ \\
\hline Triple: Gabapentinoids + NSAIDS + Tramadol & $22(6.1)$ \\
\hline Quadruple: Gabapentinoids + NSAIDS + Tramadol + topical agents & $18(5)$ \\
\hline \multicolumn{2}{|l|}{ Medication dispensing } \\
\hline Prescription (orthopedist, neurologist, neurosurgeon) & $300(83.5)$ \\
\hline No prescription & $60(16.5)$ \\
\hline \multicolumn{2}{|l|}{ Medication utilization duration } \\
\hline$<1$ month & $40(11.1)$ \\
\hline$<6$ months & $132(36.7)$ \\
\hline 6 months -1 year & $94(26.1)$ \\
\hline$>1$ year & $94(26.1)$ \\
\hline
\end{tabular}

NSAIDs nonsteroidal anti-inflammatory drugs, SD Standard deviation 


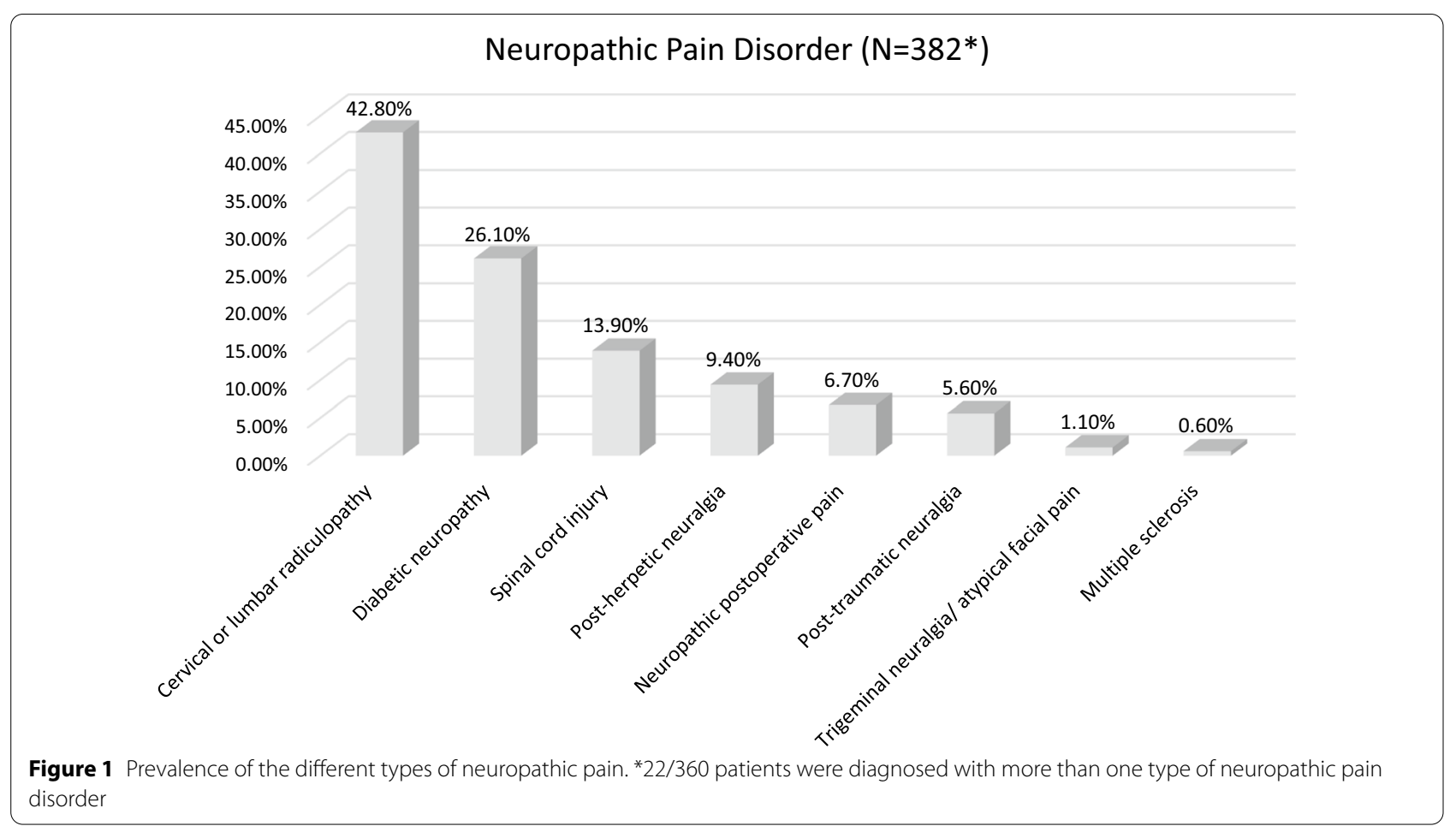

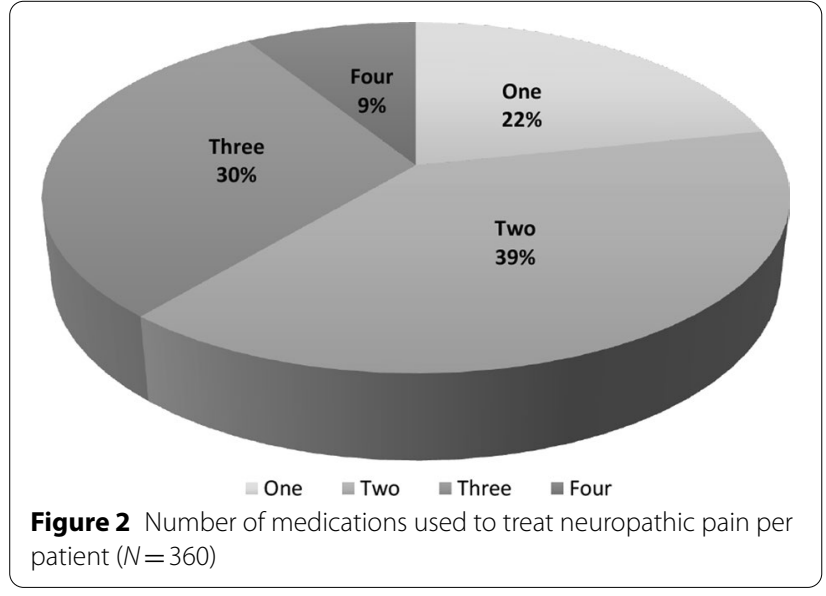

of enrolled patients were taking guideline-recommended first-line agents. Contrarily, a recent French study assessing general physicians' extent of adherence to guidelines detected their obliviousness of first-line options [14].

Post-herpetic neuralgia was the most common etiology treated without any prescription. This can be attributed to the fact that many people seek advice and treatment from the community pharmacy to diagnose and manage skin conditions, including herpes zoster and its complications [15].
Gabapentinoids, mainly pregabalin, were the most commonly used drugs to treat neuropathic pain from different etiologies. While having a comparable mechanism of action, pregabalin has a higher analgesic potency than gabapentin, due to its higher binding affinity for the alpha-2/delta-1 subunit and higher bioavailability [16]. Pregabalin's specific pharmacokinetic benefits over gabapentin may have been the basis for its popularity among prescribing doctors.

In contrast, only a minority of patients used SNRIs and TCAs in the present study. The transition from TCAs to gabapentinoids can be attributed mainly to their low safety profile, especially in the elderly population as they can cause a broad range of adverse effects as orthostatic hypotension, xerostomia, and constipation. Secondary amines (nortriptyline or desipramine) has been associated with more minor anticholinergic effects and sedation and thus are better tolerated than other nonselective TCAs (amitriptyline and imipramine) [17]. TCAs also have a multitude of contraindications, including glaucoma, prostate hypertrophy, and cardiac conduction irregularities [9]. However, TCAs may be a preferred option for patients with severe renal insufficiency, given their hepatic elimination [9].

Surprisingly, although SNRIs have a relatively safer adverse-effects profile than TCAs (anticholinergic and cardiac side effects), their dispense rate was limited in this study. This may be attributed to the increased costs 
Table 2 Demographic and clinical factors associated with the most commonly used drugs for neuropathic pain

\begin{tabular}{lccc}
\hline Evaluated factors & $\begin{array}{l}\text { First-line agents } \\
\text { OR }(95 \% \mathbf{C l})\end{array}$ & $\begin{array}{l}\text { Opioids } \\
\text { OR (95\% Cl) }\end{array}$ & $\begin{array}{l}\text { NSAIDs } \\
\text { OR (95\% Cl) }\end{array}$ \\
\hline Age & $0.96(0.93-0.98)^{\dagger}$ & $0.98(0.95-1.01)$ & $1.03(1.01-1.05)^{\dagger}$ \\
Female (ref male) & $0.46(0.21-1.00)$ & $1.36(0.61-3.01)$ & $0.45(0.26-0.79)^{\dagger}$ \\
Diabetes mellitus & $0.75(0.24-2.32)$ & $3.38(0.69-16.58)$ & $0.75(0.24-2.32)$ \\
Anxiety & $0.34(0.09-1.27)$ & $1.00(0.29-3.38)$ & $0.67(0.26-1.69)$ \\
Depression & $1.16(0.47-2.89)$ & $1.00(0.41-2.44)$ & $0.99(0.53-1.82)$ \\
Alcohol intake & $0.41(0.19-0.90)^{\dagger}$ & $2.32(1.01-5.35)^{\dagger}$ & $0.96(0.52-1.77)$ \\
Smoking status & $0.89(0.42-1.86)$ & $3.59(1.46-8.81)^{\dagger}$ & $0.56(0.32-1.00)$ \\
Diabetic neuropathy & $6.27(1.72-22.82)^{\dagger}$ & $0.05(0.004-0.62)^{\dagger}$ & $1.09(0.25-4.75)$ \\
Post-herpetic neuralgia & $6.15(0.78-47.99)$ & $0.14(0.01-1.57)$ & $1.31(0.33-5.20)$ \\
Cervical/lumbar radiculopathy & $2.74(0.46-16.16)$ & $0.05(0.008-0.34)^{\dagger}$ & $2.63(0.97-7.12)$ \\
Spinal cord injury & $3.00(0.46-19.26)$ & $0.99(0.16-6.11)$ & $2.33(0.74-7.29)$ \\
Neuropathic post-operative pain & $1.78(0.24-12.86)$ & $0.79(0.12-5.14)$ & $1.01(0.28-3.61)$ \\
Trigeminal neuralgia & $0.55(0.05-7.50)$ & $0.75(0.04-11.60)$ & $0.00(0.00-)$ \\
Post traumatic neuralgia & $1.02(0.99-5.57)$ & $1.14(0.15-8.27)$ & $1.20(0.30-4.79)$ \\
Prescribed by a physician & $23.33(9.36-58.11)^{\dagger}$ & $5.17(1.02-25.95)^{\dagger}$ & $0.10(0.03-0.26)^{\dagger}$ \\
\hline
\end{tabular}

$C /$ confidence interval, NSAIDs nonsteroidal anti-inflammatory drugs, $O R$ odds ratio ${ }^{\dagger} p<0.05$

of SNRI compared to other first-line options in addition to the CYP2D6 inhibition and worsened glycemic control precipitated by duloxetine [9]. In general, these considerations may drive the Lebanese community's preference for pregabalin over TCAs and SNRIs. In addition, gabapentinoids monotherapy is as effective as amitriptyline with better tolerable adverse effects [18]. Many studies supported the use of pregabalin as a first-line treatment in neuropathy and its efficacy in reducing pain, according to several types of pain assessment scores and improving sleep quality [19]. Sleep hygiene should be regarded as an important therapeutic target as insomnia significantly impacts pain severity.

Our findings point to significant concerns about particular combinations and the use of NSAIDs for treating neuropathic pain. Although the majority of patients were taking a combination of drugs, which is consistent with previous research showing that at least $45 \%$ of patients with neuropathic pain are treated with two or more drugs, the most common combinations encountered were gabapentinoid plus NSAID, gabapentinoid plus tramadol, and gabapentinoid plus NSAID plus tramadol [6]. It is noteworthy that we did not find any combination of the two first-line drugs recommended by NeuPSIG (for example, gabapentinoid plus TCA or SNRI) in case of inadequate relief with a single agent [5]. There is no clear agreement on the best next step in case of therapy failure; whether to alternate between same class or different class medications or even to add a particular new drug. However, the US Food and Drug Administration has expressed concern over the increasing use of gabapentinoids, especially when administered concurrently with opioid analgesics or benzodiazepines, due to increased risk of potential harm predisposed by drug interactions and accented adverse events [20]. An additional problem arises in the Lebanese community, where current governmental regulations are insufficient to control the dispensation of gabapentinoids, as confirmed by $43 \%$ of Lebanese pharmacists [13].

Thus, no surprise that the elderly received less prescriptions of first-line medications given the vulnerable safety profile of these drugs. Moreover, the decreased dispense of opioids amongst PDN and cervical or lumbar radiculopathy patients may be attributed to the lack of evidence regarding the role of opioids as a first line option for patients with DPN and their limited use in neuropathic back pain for a duration up to 2 months [4$7,21]$. However, the direct association between positive alcohol intake and smoking history is unsound as the use of opioid predisposes a grave issue due to the increased risk of overdose.

The second concern is the overuse of NSAIDs (diclofenac, naproxen, and ibuprofen) to provide additional pain relief in NeP disorders in the Lebanese community, despite the lack of solid evidence that proves their use. The continued widespread use of NSAIDs for neuropathic pain was also reported by Moore and colleagues, where an estimated 18 to $47 \%$ of $\mathrm{NeP}$ patients declared the use of NSAIDs specifically for their neuropathic pain [8]. Notably, in our current analysis, 
physicians visit was not directly associated with NSAIDs use. This directs the blame away from prescribing doctors and condemns patients and dispensing pharmacists given the over-the-counter dispensary of NSAIDs. In fact, selfmedication has been widely detected in the Lebanese community, as reported by Awada and colleagues, where acetaminophen-based analgesics (48.7\%) and NSAIDS (24.6\%) were the most commonly consumed medications [22]. Thus, a strategy must be developed to implement influential community pharmacy-based interventions to raise patient and pharmacist awareness about the rational use of drugs for neuropathy.

To our knowledge, this is the first study that describes the patterns of pain medication dispensing in neuropathic diseases in Lebanon. Nevertheless, it was subject to some limitations. First, it is an observational study in which outcomes were not evaluated. Second, not all Lebanese pharmacies were included in the sample studied. Third, data were reported by the patient and may be subject to recall bias.

\section{Conclusions}

In Lebanon, neuropathic pain management comply with the international NeuPSIG guideline. Pregabalin is the most commonly used drug, and combination treatments are usually needed to alleviate neuropathic pain.

\begin{abstract}
Abbreviations
AAN: American Academy of Neurology; APS: American Pain Society; CPS: Canadian Pain Society; EFNS: European Federation of Neurological Sciences; IASP: International Association of the Study of Pain; NeP: Neuropathic pain; NeuPSIG: Neuropathic Pain Special Interest Group; NSAIDs: Nonsteroidal antiinflammatory drugs; SNRIs: Serotonin-norepinephrine reuptake inhibitors; SD: Standard deviation; SPSS: Statistical Package for the Social Sciences; TCAs: Tricyclic antidepressants.
\end{abstract}

\section{Acknowledgements}

Not applicable.

\section{Authors' contributions}

$\mathrm{BC}$ developed the research questionnaire, study design and performed the statistical analyses. BC and FS acquired the data, drafted the manuscript, and revised it critically for important intellectual content. Both the authors read and approved the final manuscript

\section{Funding}

No sources of funding were used in the preparation of this study.

\section{Availability of data and materials}

The data sets generated during and/or analyzed during the current study are available from the corresponding author on reasonable request.

\section{Declarations}

\section{Ethics approval and consent to participate}

This study was approved by the ethics committee of Lebanese International University, School of Pharmacy with approval number (Ref:2018/10). The participants provided verbal consent, because this was an observational study involving a patient interview. No interventions were applied on the participants, and the institution review board approved the study based on this verbal consent.

\section{Consent for publication}

Not applicable.

\section{Competing interests}

The authors declare that they have no competing interests.

Received: 21 July 2021 Accepted: 15 October 2021

Published online: 26 October 2021

\section{References}

1. Neuropathic pain in adults: pharmacological management in non-specialist settings. London: National Institute for Health and Care Excellence (UK). 2020. https://www.nice.org.uk/guidance/cg173.

2. Bouhassira D. Neuropathic pain: definition, assessment and epidemiology. Rev Neurol (Paris). 2019;175(1-2):16-25. https://doi.org/10.1016/j. neurol.2018.09.016.

3. Cavalli E, Mammana S, Nicoletti F, Bramanti P, Mazzon E. The neuropathic pain: an overview of the current treatment and future therapeutic approaches. Int J Immunopathol Pharmacol. 2019. https://doi.org/10. 1177/2058738419838383

4. Dworkin RH, O'Connor AB, Kent J, Mackey SC, Raja SN, Stacey BR, et al. Interventional management of neuropathic pain: NeuPSIG recommendations. Pain. 2013;154(11):2249-61. https://doi.org/10.1016/j.pain.2013.06. 004.

5. Cruccu G, Truini A. A review of neuropathic pain: from guidelines to clinical practice. Pain Therapy. 2017;6(Suppl 1):35-42. https://doi.org/10.1007/ s40122-017-0087-0.

6. Moulin D, Boulanger A, Clark AJ, Clarke H, Dao T, Finley GA, et al. Pharmacological management of chronic neuropathic pain: revised consensus statement from the Canadian Pain Society. Pain Res Manag. 2014;19(6):328-35. https://doi.org/10.1155/2014/754693.

7. Attal N, Cruccu G, Baron R, Haanpää M, Hansson P, Jensen TS, et al. EFNS guidelines on the pharmacological treatment of neuropathic pain: 2010 revision. Eur J Neurol. 2010;17(9):1113-e88. https://doi.org/10.1111/j. 1468-1331.2010.02999.x.

8. Moore RA, Chi CC, Wiffen PJ, Derry S, Rice AS. Oral nonsteroidal antiinflammatory drugs for neuropathic pain. Cochrane Database Syst Rev. 2015;2015(10): CD010902. https://doi.org/10.1002/14651858.CD010902. pub2.

9. Bohlega S, Alsaadi T, Amir A, Hosny H, Karawagh AM, Moulin D, et al. Guidelines for the pharmacological treatment of peripheral neuropathic pain: expert panel recommendations for the middle East region. J Int Med Res. 2010;38(2):295-317. https://doi.org/10.1177/147323001003800 201.

10. Zeitoun AA, Dimassi HI, Chami BA, Chamoun NR. Acute pain management and assessment: are guidelines being implemented in developing countries (Lebanon). J Eval Clin Pract. 2013;19(5):833-9. https://doi.org/ 10.1111/j.1365-2753.2012.01860.x.

11. Gordon DB, Dahl JL, Miaskowski C, McCarberg B, Todd KH, Paice JA, et al. American pain society recommendations for improving the quality of acute and cancer pain management: American Pain Society Quality of Care Task Force. Arch Intern Med. 2005;165(14):1574-80. https://doi.org/ 10.1001/archinte.165.14.1574.

12. Centers for disease control and prevention. Epi info 7 available on https:// www.cdc.gov/epiinfo/index.html. Accessed 5 Jan 2019.

13. Tarhini F, Taky R, Jaffal LH, Kresht J, Elchaer G, Awada S, et al. Awareness of Lebanese pharmacists towards the use and misuse of gabapentinoids and tramadol: a cross-sectional survey. Dr Sulaiman Al Habib Med J. 2020;2(1):24. https://doi.org/10.2991/dsahmj.k.200206.002.

14. Martinez V, Attal N, Vanzo B, Vicaut E, Gautier JM, Bouhassira D, et al. Adherence of French GPs to chronic neuropathic pain clinical guidelines: results of a cross-sectional, randomized, "e" case-vignette survey. PLoS ONE. 2014:9(4): e93855. https://doi.org/10.1371/journal.pone.0093855.

15. Dumpit JR, McGuire CD, Wang FY, Crandall B, Chen TC. Evaluation of a pharmacist managed telephone pain clinic for neuropathy treatment. 
J Pain Palliat Care Pharmacother. 2020;34(4):219-24. https://doi.org/10. 1080/15360288.2020.1814479.

16. Bockbrader HN, Wesche D, Miller R, Chapel S, Janiczek N, Burger P. A comparison of the pharmacokinetics and pharmacodynamics of pregabalin and gabapentin. Clin Pharmacokinet. 2010;49(10):661-9. https://doi.org/ 10.2165/11536200-000000000-00000.

17. Watson CP, Vernich L, Chipman M, Reed K. Nortriptyline versus amitriptyline in postherpetic neuralgia: a randomized trial. Neurology. 1998:51(4):1166-71. https://doi.org/10.1212/wnl.51.4.1166.

18. Moore RA, Wiffen PJ, Derry S, McQuay HJ. Gabapentin for chronic neuropathic pain and fibromyalgia in adults. Cochrane Database Syst Rev. 2011 https://doi.org/10.1002/14651858.CD007938.pub2.

19. Taguchi T, Nozawa K, Parsons B, Yoshiyama T, Ebata N, Igarashi A, et al. Effectiveness of pregabalin for treatment of chronic cervical radiculopathy with upper limb radiating pain: an 8-week, multicenter prospective observational study in Japanese primary care settings. J Pain Res. 2019;12:1411-24. https://doi.org/10.2147/JPR.S191906.

20. Johansen ME. Gabapentinoid use in the United States 2002 through 2015. JAMA Intern Med. 2018;178(2):292-4. https://doi.org/10.1001/jamai nternmed.2017.7856.

21. Eisenberg E, McNicol E, Carr DB. Opioids for neuropathic pain. Cochrane Database Syst Rev. 2006. https://doi.org/10.1002/14651858.CD006146.

22. Awada S, Diab B, Khachman D, Zeidan RK, Slim H, Zein S, et al. Selfmedication practices and knowledge among Lebanese population: a cross-sectional study. Dr Sulaiman Al Habib Med J. 2020;2(2):56.

\section{Publisher's Note}

Springer Nature remains neutral with regard to jurisdictional claims in published maps and institutional affiliations.

\section{Submit your manuscript to a SpringerOpen ${ }^{\circ}$ journal and benefit from:}

- Convenient online submission

- Rigorous peer review

- Open access: articles freely available online

- High visibility within the field

- Retaining the copyright to your article

Submit your next manuscript at $\boldsymbol{\nabla}$ springeropen.com 